\section{Chrischta Ganz}

Im April dieses Jahres fand in Arlesheim die Weiterbildung «Natura Zyklus Fokus» zum Thema «Tumorerkrankungen - anthroposophische Gesichtspunkte zu deren Entstehung und adjuvanten Behandlung» statt. Dabei wurden die Tumorentstehung, die verschiedenen Mistelpräparate sowie mögliche adjuvante Begleittherapien betrachtet.

Referenten waren:

- René Schwarz, Naturheilpraktiker, Dozent für Phytotherapie, «zusammenhänge $\mathrm{GmbH}-G e s u n d h e i t s-$ förderung natürlich»;

- Dr. rer. nat. Hartmut Ramm, Botanische Abteilung Institut Hiscia, Verein für Krebsforschung;

- Dr. med. Kaspar Jaggi, Arzt für allgemeine und anthroposophische Medizin in Arztpraxis in Jegensdorf, Medizinisch-Wissenschaftliche Abteilung Weleda AG;

- Dr. med. Clifford Kunz, Facharzt für Innere Medizin FMH, Ita Wegman Ambulatorium Basel;

- Dr. rer. nat. Michael Werner, Leitung Institut Hiscia, Verein für Krebsforschung.

«Natura Zyklus Fokus» ist ein Weiterbildungsangebot, das gemeinsam von «zusammenhänge GmbH» mit der Saner Apotheke AG durchgeführt wird. Die Saner Apotheke ist Initiator und übernimmt die Organisation. Verantwortliche sind:

- Dominik Saner, Offizinapotheker FPH, Naturarzt, Inhaber Saner Apotheke AG;

- Edith Ehrsam, Apothekerin FPH, stellvertretende Geschäftsführerin Saner Apotheke Basel Markthalle, Leitung Weiterbildung.

«zusammenhänge $\mathrm{GmbH}$ », ein Netzwerk von Naturheilpraktikern

\title{
Weiterbildung «Natura Zyklus Fokus»: Nachbericht
}

und Therapeuten, setzt sich für eine qualitativ hochstehende Naturheilkunde ein und erarbeitet praxisorientierte und zeitgemässe Konzepte zum Thema Naturheilkunde. Für medizinische Fachpersonen wird neben «Natura Zyklus Fokus» auch «Natura Zyklus Basis» zum Thema «Einstieg in eine anthroposophisch orientierte Naturheilkunde» (Juni/Juli 2014) angeboten.

Dr. med. Kaspar Jaggi startete in seinem Referat «Naturheilkundliche Betrachtungen zur Tumorentstehung» mit der Frage, was Krebs ist. Fest steht, dass die Diagnose eines Tumors grosse Betroffenheit und Verunsicherung beim Patienten auslöst. Die Frage «Warum gerade ich?» fordert den erkrankten Menschen auf, sich mit grossen Lebensfragen und der eigenen Vergänglichkeit auseinanderzusetzen. Hierbei ist essenziell, wie der behandelnde Arzt das Krebsgeschehen betrachtet. Ist eine Tumorerkrankung ein (genetisches) Zellteilungsproblem? Oder ist sie eine Systemerkrankung, da sich der Körper nicht mehr gegen maligne Zellbildung wehrt? Fällt die Antwort pathogenetisch aus? Oder steht die Resilienz im Vordergrund, wie es die Erkenntnisse des Krebsspezialisten Dr. O. Carl Simonton aufzeigen? Hält man sich vor Augen, wie stark die Angst vor Krebs (unabhängig davon, ob ein Tumor diagnostiziert ist oder nicht) die Immunabwehr schwächt, sollten salutogentische Aspekte in jeder Krebsprävention/-therapie an erster Stelle stehen.

Trotz der enormen Errungenschaften der modernen Medizin (insbesondere der Aufschlüsselung der Genome) konnten zum Tumorgeschehen

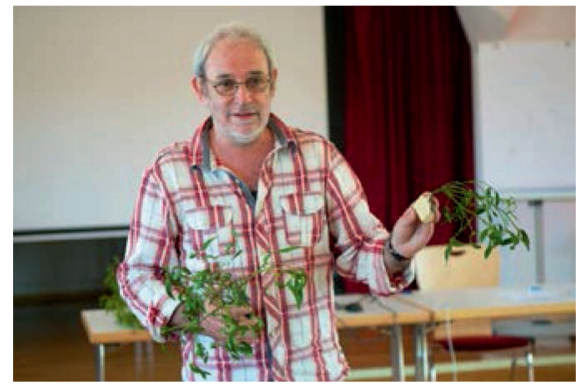

Abb. 1. René Schwarz stellte die Heilpflanze Mistel vor (Bild: Marc Gilgen).

jedoch lediglich Teilantworten gefunden werden. Aus ganzheitlicher Sicht ist ein Tumor immer multifaktoriell bedingt und Ausdruck und nicht Ursache einer Erkrankung. Die Anthroposophie sieht im Tumor eine Störung im Gesamtwesen, die das unkontrollierte Wachstum erst ermöglicht. Die Formgesetzmässigkeiten und die Kommunikation zwischen malignen und gesunden Zellen sind gestört.

Dr. med. Kaspar Jaggi nahm weiter Bezug auf Aussagen von John Beard [1]. Er beschreibt die Ähnlichkeit von Krebszellen mit trophoblastischen Embryonalzellen, die zu Beginn einer Schwangerschaft das Einnisten des Embryos ermöglichen und aggressivchaotisch wachsen. Die sich schnell teilenden Zellen gewinnen ihre Energie aus der Zuckergärung, unterdrücken das mütterliche Immunsystem und produzieren humanes Choriongonadotropin (hCG; heute als Tumormarker anerkannt). Die Wucherung stoppt erst, wenn der Embryo ab der siebten Woche Pankreasenzyme erzeugt.

Im Anschluss folgte das Referat «Pharmazeutische Betrachtungen zur Mistel sowie die Verarbeitungsprozesse der Mistel» des Naturheilpraktikers René Schwarz (Abb. 1), der viel

\section{KARGER}

Fax +4976145207 14 (c) 2014 S. Karger GmbH, Freiburg
Chrischta Ganz

Praxis für Naturheilkunde

Friedhofstrasse 15, 8636 Wald ZH, Schweiz

naturheilpraxis@chrischtaganz.ch www.chrischtaganz.ch 


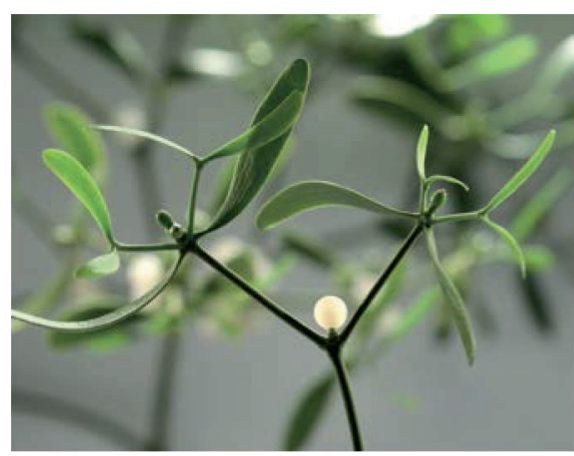

Abb. 2. Mistel, die aussergewöhnliche Heilpflanze (Bild: Jürg Buess).

Erfahrung aus seiner langjährigen Tätigkeit bei führenden komplementärmedizinischen Arzneimittelanbietern mitbringt, und Dr. rer. nat. Hartmut Ramm, der seit 27 Jahren mit der Mistel arbeitet - sowohl als Gärtner als auch in der Mistelforschung.

Die enge Beziehung von Dr. Hartmut Ramm zur Mistel sowie sein grosses Wissen wurden im Referat sichtbar, als er die Mistel als eine uralte Heilpflanze mit ihrer im Pflanzenreich aussergewöhnlichen Lebensform vorstellte (Abb. 2).

Die Mistel bildet keine Wurzeln, sondern ist für ihre Ernährung auf den Wirtsbaum angewiesen. Auch fehlen den Mistelblättern die üblichen Strukturen, die für eine optimale Photosynthese nötig sind, und die Entwicklung der pflanzentypischen Strukturen wie Blätter und Stängel ist verzögert bis reduziert. Die Mistel bildet Mistellektine anstatt Wurzeln sowie Viscotoxine anstelle der Verholzung und der Blätter.

In der keltischen Tradition galt die Mistel, insbesondere die Eichenmistel, als heilige Pflanze (Mistel $=$ «die alles Heilende» [1]). In der Erntezeremonie wurden Sonne und Mond von zwei Druiden symbolisiert. Ein weissgekleideter Druide, Stellvertreter für Aktivität, die solaren Kräfte und den Blick «nach aussen», schnitt die Mistel mit einer goldenen Sichel, die von einem weissen Tuch aufgefangen wurde, damit sie den Boden nicht berühre. Ein zweiter Druide, ganz in schwarz gekleidet, symbolisierte die lunaren Kräfte, Ruhe und Verinnerlichung. Die Polarität von Aktivität und Ruhe, von Sonne und Mond wurde so rituell «gefeiert».

Basierend auf diesen Betrachtungen wird für anthroposophische Mistelpräparate die Sommer- und Winterernte im Herstellungsprozess vereinigt. Aus heutiger Sicht ist das besonders interessant, da die höchste Konzentration der Mistellektine im Winter (im Inneren der Mistelpflanze) und die höchste Konzentrationen der Viscotoxine im Sommer (in der Peripherie der Mistel) nachgewiesen werden konnten. Im Jahreslauf zeigen sich diese räumlich-zeitlichen Gegensätze in korrespondierender Weise.

Die Mistel ist nicht nur philosophisch gesehen noch nie auf der Erde gewesen. Sie ist auf Vögel angewiesen, um ihren Samen verfügbar zu machen. Erst wenn der Vogel die Beere verschluckt und mit seinem Kot wieder ausgeschieden hat, verliert der Samen seine erdferne Existenz und tritt mit dem Baum in Kontakt. Auch die Wachstumsrichtung ist umgekehrt als üblicherweise bei Pflanzen, die Mistel differenziert nicht aus und hat keinen Sinn für Schwerkraft. Steiner bezeichnete diese Besonderheiten als «Antitendenz». Die Mistel erhält veränderte Erdensubstanz durch den Baum, d.h., der Baum selektiert die Säfte, die bei der Mistel ankommen, ähnlich wie der Körper einer schwangeren Frau den Embryo ernährt. Die Mistel ist damit nicht nur bodenfern, sondern trägt auch die Qualität des «Ungeborenen» in sich und der Bezug zu Beards Aussage über die trophoblastischen Embryonalzellen wird sichtbar.

Im Anschluss an diese «Begegnung» mit der Mistel zeigte René Schwarz die Unterschiede der verschiedenen Mistelpräparate auf. Die von Rudolf Steiner veröffentlichten Herstellungsbedingungen (Sommerernte/Winterernte, Mistelsaft in vertikaler Bewegung, Durchsetzen von einer horizontal rotierenden Bewegung, Mistelsaft tropft und wird in Tropfen durchkreist, Wiederverbinden in Horizontalkreisen mit Mistelsaft) werden alle in den bekanntesten Mistel-Krebstherapeutika umgesetzt [1]. Ansonsten gibt es einige Unterschiede, die dem verordnenden Arzt ebenso bekannt sein müssen wie die unterschiedliche Verträglichkeit je nach Wirtsbaum (Nadelhölzer oder Laubbäume) und Präparat bzw. Hersteller.

Auch die therapeutische Breite, Kontraindikation sowie die Kombination mit onkologischen Therapien wurden von René Schwarz sowie von Dr. med. Clifford Kunz im nachfolgenden Referat «Tumorbehandlung aus anthroposophischer Sicht - Wissenswertes aus der Sicht eines verordnenden Arztes» aufgezeigt.

Die intensive Beziehung der Mistel zum Licht sowie ihre Symbolkraft für Geduld und Reduktion auf das Wesentliche (nicht $\mathrm{zu}$ verwechseln mit Reduktion auf das Minimum) ermöglichen dem erkrankten Menschen, seine «leibliche Identität» zu erhalten bzw. wieder zu finden.

Dr. med. Clifford Kunz betonte im Weiteren, wie wichtig das psychische Gleichgewicht, die Stärkung von Regeneration, die Regulation von Entgiftung und Immunabwehr, die spirituellen Ressourcen, das Einhalten circadianer Rhythmen, eine spezifische Ernährung sowie die körperliche Bewegung für Therapie und Heilungsverlauf sind.

Der äusserst gelungene Weiterbildungstag schloss mit einer Betriebsführung des Instituts Hiscia, wo Dr. rer. nat. Michael Werner den Kursteilnehmern einen Einblick in die Herstellung des Mistelpräparats Iscador bot.

\section{Literatur}

1 Kursunterlagen Natura Zyklus Fokus, April 2014. 